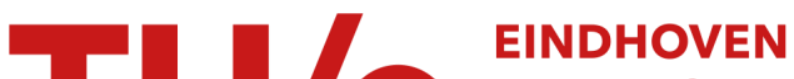 \\ UNIVERSITY OF \\ TECHNOLOGY
}

\section{Low-energy-spread ion bunches from a trapped atomic gas}

\section{Citation for published version (APA):}

Reijnders, M. P., Kruisbergen, van, P. A., Taban, G., Geer, van der, S. B., Mutsaers, P. H. A., Vredenbregt, E. J. D., \& Luiten, O. J. (2009). Low-energy-spread ion bunches from a trapped atomic gas. Physical Review Letters, 102(3), 034802-1/4. [034802]. https://doi.org/10.1103/PhysRevLett.102.034802, https://doi.org/10.1103/PhysRevLett.102.034802

DOI:

10.1103/PhysRevLett.102.034802

10.1103/PhysRevLett.102.034802

Document status and date:

Published: 01/01/2009

\section{Document Version:}

Publisher's PDF, also known as Version of Record (includes final page, issue and volume numbers)

\section{Please check the document version of this publication:}

- A submitted manuscript is the version of the article upon submission and before peer-review. There can be important differences between the submitted version and the official published version of record. People interested in the research are advised to contact the author for the final version of the publication, or visit the $\mathrm{DOI}$ to the publisher's website.

- The final author version and the galley proof are versions of the publication after peer review.

- The final published version features the final layout of the paper including the volume, issue and page numbers.

Link to publication

\section{General rights}

Copyright and moral rights for the publications made accessible in the public portal are retained by the authors and/or other copyright owners and it is a condition of accessing publications that users recognise and abide by the legal requirements associated with these rights.

- Users may download and print one copy of any publication from the public portal for the purpose of private study or research.

- You may not further distribute the material or use it for any profit-making activity or commercial gain

- You may freely distribute the URL identifying the publication in the public portal.

If the publication is distributed under the terms of Article 25fa of the Dutch Copyright Act, indicated by the "Taverne" license above, please follow below link for the End User Agreement:

www.tue.nl/taverne

Take down policy

If you believe that this document breaches copyright please contact us at:

openaccess@tue.nl

providing details and we will investigate your claim. 


\title{
Low-Energy-Spread Ion Bunches from a Trapped Atomic Gas
}

\author{
M. P. Reijnders, P. A. van Kruisbergen, G. Taban, S. B. van der Geer, P. H. A. Mutsaers, \\ E. J. D. Vredenbregt, and O. J. Luiten* \\ Department of Applied Physics, Eindhoven University of Technology, P.O Box 513, 5600 MB Eindhoven, The Netherlands
} (Received 30 September 2008; published 22 January 2009)

\begin{abstract}
We present time-of-flight measurements of the longitudinal energy spread of pulsed ultracold ion beams, produced by near-threshold ionization of rubidium atoms captured in a magneto-optical atom trap. Well-defined pulsed beams have been produced with energies of only $1 \mathrm{eV}$ and a root-mean-square energy spread as low as $0.02 \mathrm{eV}, 2$ orders of magnitude lower than the state-of-the-art gallium liquid-metal ion source. The low energy spread is important for focused ion beam technology because it enables milling and ion-beam-induced deposition at sub-nm length scales with many ionic species, both light and heavy. In addition, we show that the slowly moving, low-energy-spread ion bunches are ideal for studying intricate space charge effects in pulsed beams. As an example, we present a detailed study of the transition from space charge dominated dynamics to ballistic motion.
\end{abstract}

DOI: 10.1103/PhysRevLett.102.034802

The importance of focused ion beam (FIB) applications for nanotechnology can hardly be overstated. FIBs are used for structuring at the nanometer scale both by accurate removal of material by sputtering and by high precision ion-beam-induced deposition. For this reason FIBs have become indispensable tools for circuit editing in the semiconductor industry [1]. In addition FIBs enable determination of the elemental, isotopal, and molecular composition of surfaces using time-of-flight secondary ion mass spectrometry (TOF SIMS), making TOF SIMS one of the most sensitive surface analysis techniques [2].

FIB performance is presently limited by the properties of the commonly preferred liquid metal ion source (LMIS). The gallium-based LMIS FIB offers the highest spatial resolution. The smallest focal spot size of $\sim 10 \mathrm{~nm}$, is limited by the energy spread of the $\mathrm{Ga}^{+}$source [1]. However, to keep up with Moore's law, the semiconductor industry will soon require milling and deposition at the $1 \mathrm{~nm}$ scale. In addition, for TOF SIMS heavier elements, like cesium, are preferred for their higher sputter yield. In particular the use of TOF SIMS in the life sciences requires projectiles heavier than Ga [3]. Unfortunately this goes at the expense of spatial resolution, which can only be reached at present with the Ga-based LMIS FIB.

Recently a new ion source was proposed, the Ultra Cold Ion Source (UCIS), which is based on extraction of ions from a laser cooled atomic gas [4-6]. The UCIS promises a much smaller energy spread than the LMIS at comparable brightness, enabling $\leq 1 \mathrm{~nm}$ focal spot sizes. In addition the UCIS is suited for many elements, in particular the alkali-metal atoms: Cs for TOF SIMS with high spatial resolution and high sputter yield; $\mathrm{Li}$ as an alternative for $\mathrm{He}$ in the recently developed ion microscope [7].

After first preliminary experiments [8], the UCIS potential of delivering beams of LMIS brightness was recently demonstrated [9]. In this Letter we present detailed TOF
PACS numbers: 29.27.Bd, 32.80.Fb, 41.75.Ak, 67.85.-d

energy spread measurements on rubidium ion bunches extracted from a UCIS. We have produced $\mathrm{Rb}^{+}$ion beams with a root-mean-squared (rms) energy spread of only $0.02 \mathrm{eV}, 2$ orders of magnitude smaller than the state-ofthe-art Ga-based LMIS. These extremely low-energy spreads have been realized by extracting ion bunches at energies as low as $1 \mathrm{eV}$. Since energy spread can be conserved during further acceleration, chromatic aberration can be virtually eliminated at beam energies typical for FIB operation, i.e., $30 \mathrm{keV}$. In combination with the recently measured brightness of such beams [9], this implies that sub-nm focal spot sizes are within reach for $\mathrm{Li}^{+}, \mathrm{Cs}^{+}$ and many other ionic species.

The extreme degree of control over beam properties afforded by UCISs opens up completely new applications as well. First, ultralow beam energies with meV energy spread and nm spatial resolution allow direct ion deposition, highly controlled excitation of localized surface states, and precision surface chemistry, all without sputter damage.

Second, the UCIS in pulsed mode opens up new regimes of space charge dominated beams at very low velocities. As we show in this Letter, this allows investigation of intricate space charge effects in slow motion (ion velocities of $\sim 10^{3} \mathrm{~m} / \mathrm{s}$ ) and therefore in great detail. As an example, we have studied the transition from space charge dominated dynamics to ballistic motion. Such a model system is of great importance for the study of ill-understood beam phenomena like space charge-induced instabilities, emittance growth and halo formation. These nonlinear space charge effects play a decisive role in the beam formation for the most demanding applications such as heavy ion fusion, high-energy colliders, and free electron lasers. The only system at the moment fully dedicated to the study of such space charge effects is UMER [10,11], which employs a $10 \mathrm{keV}$ electron beam in a ring. 
The basis of a UCIS is a laser cooled and trapped atomic gas inside a magneto-optical trap (MOT), which is ionized by near-threshold photoionization. By applying an electric field a cold ion beam is created, as is schematically illustrated in Fig. 1. The thermal energy of these ions $\left(100 \mu \mathrm{K} \sim 10^{-8} \mathrm{eV}\right)$ is very low, giving rise to a very high brightness. An additional longitudinal energy spread is introduced due to the initial longitudinal size of the ionization volume. A more detailed description of the source and its expected performance is given in our previous paper [4].

In our experiments we use ${ }^{85} \mathrm{Rb}$ in a standard vapor cell MOT configuration [12], which consists of 3 orthogonal pairs of circularly polarized $780 \mathrm{~nm}$ laser beams. A quadrupole magnetic field with a gradient of $10 \mathrm{G} / \mathrm{cm}$ is produced by two coils in anti-Helmholtz configuration placed around the accelerator inside the vacuum chamber. Typically $10^{7}-10^{8} \mathrm{Rb}$ atoms are captured in a cloud with a Gaussian density distribution with an rms radius of $1 \mathrm{~mm}$ and a central density of $10^{9}-10^{10}$ atoms $/ \mathrm{cm}^{3}$. To produce ions a two step photo-ionization scheme is used. First $\mathrm{Rb}$ atoms are excited to the $5 p$ level by the trapping laser, from where the atoms are ionized by a $2.5 \mathrm{~ns} \mathrm{rms}, 480 \mathrm{~nm}$ pulsed dye laser tuned just above the ionization threshold. The ionization laser beam is focused through the cold atom cloud in a direction perpendicular to the electric field (see Fig. 1). In this way a cylinder of charged particles is created with an rms radius $\sigma_{R}=32 \pm 1 \mu \mathrm{m}$.

dc voltages up to $V_{A}=5 \mathrm{kV}$ are applied across the electrodes, which are approximately $20 \mathrm{~mm}$ apart. For technical details of the accelerator structure, see Ref. [13]. The quadrupole magnetic field is kept on, but this has a neglectable effect on the trajectories. Charged particles are created at the center of the acceleration region, in a local electric field $E_{0}=V_{A} / d_{\text {eff }}$, where $d_{\text {eff }}=27 \mathrm{~mm}$. They travel about $10 \mathrm{~mm}$ before exiting the accelerator, resulting in an energy of $U=0.49 \mathrm{eV}_{A}$. The size $\sigma_{R}$ of the ionization volume in the accelerator field $E_{0}$ gives rise to an initial rms energy spread

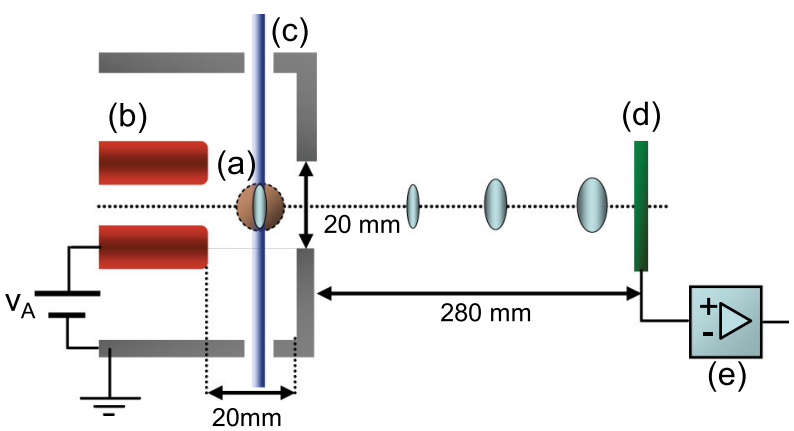

FIG. 1 (color online). A schematic overview of the experimental setup. A cold cloud of rubidium (a) is trapped inside a cylindrically symmetric accelerator structure (b). After pulsed ionization (c), ions are accelerated to the MCP detector (d) where the temporal distribution is measured (e).

$$
\sigma_{U}=e \sigma_{R} E_{0}
$$

so that $\left(\sigma_{U} / U\right)_{\text {initial }}=0.2 \%$ for $\sigma_{R}=32 \mu \mathrm{m}$.

At a distance of $29 \mathrm{~cm}$ from the accelerator center a MCP detector assembly is used to detect the ions. The temporal distribution is measured by recording the timedependent current with a transimpedance amplifier. The rms time resolution $\tau_{D}$ of the detection system is $9 \pm 1 \mathrm{~ns}$, which also includes the laser pulse length.

Time-of-flight (TOF) measurements have been performed by pulsed photo-ionization of the cold atoms in the dc acceleration field. The time-dependent current is recorded on an oscilloscope, together with a photodiode signal of the pulsed laser. The charge $Q$ in a bunch is measured with $5 \%$ accuracy by integrating the current signal. Both the photodiode signal and the current signal are fitted with a gaussian distribution to determine the average TOF $T$ and the rms bunch length $\sigma_{T}$. The relative spread in arrival time $\sigma_{T} / T$ is a measure for the longitudinal energy spread $\sigma_{U} / U$ in the bunch:

$$
\frac{\sigma_{U}}{U}=2 \frac{\sigma_{T}}{T} \frac{1}{(1-\epsilon)},
$$

where $\epsilon \approx 0.13$ is a geometric correction factor.

Measurements of the energy spread as function of the beam energy are shown in Fig. 2. Different curves correspond to different amounts of charge in a bunch, ranging from $Q=0.026 \mathrm{fC}$ to $Q=13 \mathrm{fC}$. The results shown are averages over 10 bunches. At higher voltages $\sigma_{T}$ is close to the time constant $\tau_{D}$ of the detection system, so a deconvolution is performed by quadratically subtracting $\tau_{D}$.

Two different regions can be clearly observed in the plot. At higher acceleration voltages the curves of the different

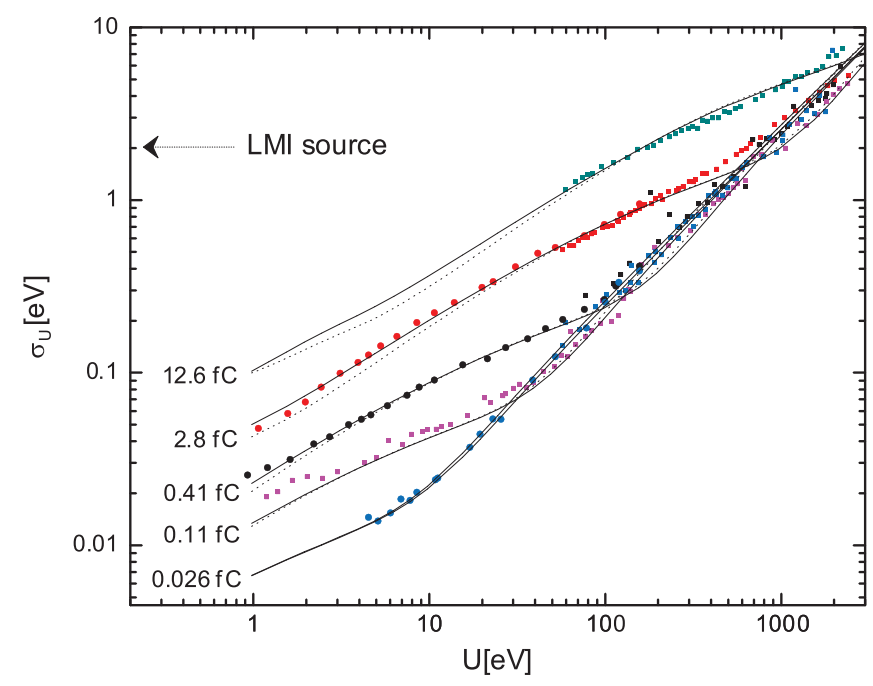

FIG. 2 (color). Measured energy spread $\sigma_{U}$ as function of the beam energy $U$ for various bunch charges (scatter plots) together with the results of the GPT simulations (dotted and solid curves). The dotted curves have been calculated directly in the GPT simulations; the solid curves have been calculated by applying the TOF procedure of Eq. (2). 
bunch charges coincide, implying that space charge forces only play a minor role. In this region the relative energy spread $\sigma_{U} / U$ is less than $0.2 \%$, as expected on the basis of Eq. (1). At lower voltages, however, the curves clearly depend on the charge in the bunch, and thus space charge is important in this region. With beam energies as low as $1 \mathrm{eV}$, this is not unexpected. At these low energies it is possible to produce beams with an rms energy spread $\sigma_{U}=0.02 \mathrm{eV}, 2$ orders of magnitude lower than the LMIS.

To get a better understanding of the behavior, particle tracking simulations have been performed with the use of the GPT code [14]. The dc electric field inside the accelerator has been calculated with the Superfish poisson solver [15]. All the ions in the bunch are tracked individually with all mutual Coulomb interactions included, using the Barnes Hutt algorithm [16] to reduce the calculation time. In Fig. 2 the simulation results are shown for five different charges. The directly calculated energy spread is indicated by dotted curves. Also the same procedure used in the experiment can be performed, by using Eq. (2) (shown as solid lines). Both are in remarkably good agreement with the experimental data, without any fit parameter.

The observed behavior can be fully explained in terms of space charge forces and acceleration fields, the only ingredients in the GPT simulation. This implies that all the data can be scaled to a single curve by dividing both the acceleration voltage and the observed energy spread by the bunch charge, as shown in Fig. 3.

The energy spread at higher acceleration voltages is dominated by the initial spread in position in the acceleration field due the size of the ionization volume, as described by Eq. (1). In this region the energy spread depends linearly on the acceleration voltage, indicated by the dashed line in Fig. 3.

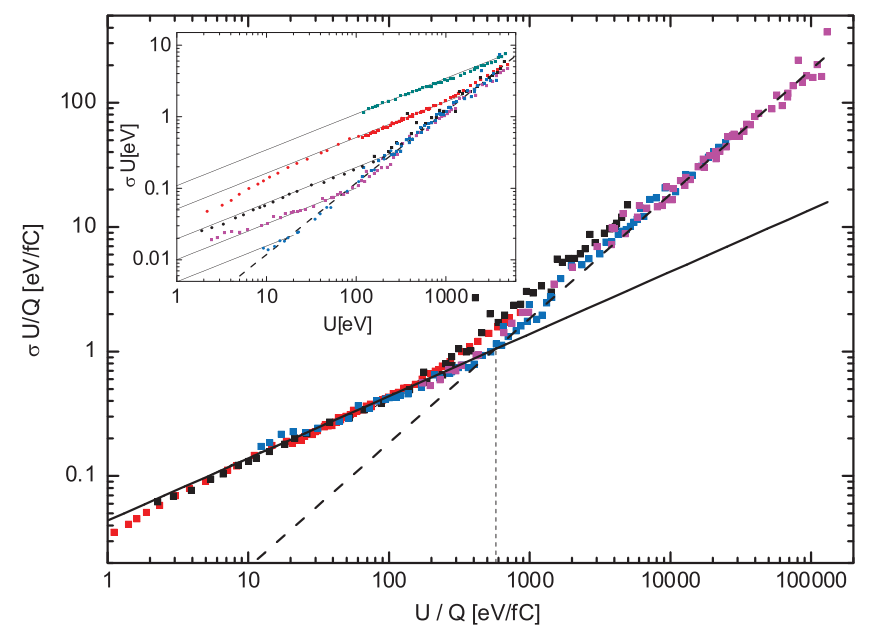

FIG. 3 (color). The five measured energy spread curves from Fig. 2 scaled by the bunch charge to a single curve. The simple analytical model given by Eq. (3) (dotted and solid lines) and Eq. (1) (dashed line) are plotted. The inset shows the data without the scaling.
At lower acceleration voltages space charge effects dominate which give rise to a square root like behavior: $\sigma_{U} \sim \sqrt{U}$. This can be understood as follows; The space charge expansion takes place on a time scale $\sim \omega_{p}^{-1}$ with $\omega_{p}=\sqrt{n_{\text {ion }} e^{2} /\left(m \epsilon_{0}\right)}$ the plasma frequency, $n_{\text {ion }}$ the initial peak ion density, $m$ the ion mass, and $\epsilon_{0}$ the permittivity of free space. In the region where the square root behavior appears, the bunches move so slowly that most of the internal (space charge) potential energy in the bunch has been converted into kinetic energy before it hits the detector. In that case for each particle the asymptotic velocity $v_{s p}$ due to the space charge expansion can be added to the velocity $v_{0}$ due to the accelerating fields. The energy spread is then given by

$$
\sigma_{U}=\frac{1}{2} m\left\langle\left(v_{0}+v_{s p}\right)^{2}-\left\langle v_{0}+v_{s p}\right\rangle^{2}\right\rangle=m \sigma_{v_{s p}}\left\langle v_{0}\right\rangle,
$$

where \langle\rangle denotes averaging over all the particles and $\sigma_{v_{s p}}$ is the rms space charge expansion velocity. Since $v_{0} \sim \sqrt{U}$ this model gives indeed the observed square root behavior.

The asymptotic space charge velocity $v_{s p}$ can be easily calculated for a uniform sphere. With a correction factor $\eta$ for the geometry this can be used to estimate the final space charge velocity for the Gaussian, cylindrically shaped bunch:

$$
\sigma_{v_{s p}}=\eta \sqrt{2 / 3} \sigma_{R} \omega_{p}
$$

In Fig. 3 the resulting model curve, obtained by combining Eq. (3) and (4) and taking $\eta=0.4$, is indicated by a dotted line. It shows good agreement with the measured data. In the inset the separate curves for the different charges, without the scaling, are plotted together with the measured data.

In the crossover region around the intersection of the dashed and the dotted lines, where both space charge forces and the initial size contribute, an interesting effect occurs for large values of $\sigma_{R}$. In Fig. $4 \sigma_{U} / U$ is plotted as a function of $U$ for $\sigma_{R}=180 \mu \mathrm{m}$ and $\sigma_{R}=32 \mu \mathrm{m}$ with, respectively, $Q \approx 0.5 \mathrm{fC}$ and $Q=0.42 \mathrm{fC}$. At high voltages we find that the relative energy spread goes to a constant value [Eq. (1)], which can indeed be controlled by changing the size of the ionization volume. This implies that even much lower relative energy spreads can be achieved than reported in this Letter by further reduction of the ionization laser beam waist. At the lowest voltages again space charge dominates, so $\sigma_{U} / U \propto U^{-1 / 2}$.

In the crossover region $(10 \mathrm{eV}<U<100 \mathrm{eV})$, however, the relative energy spread for $\sigma_{R}=180 \mu \mathrm{m}$ is significantly lower than the asymptotic high-voltage value. In this region space charge forces apparently give rise to a reduction of the relative energy spread. This counterintuitive effect can be understood as follows: in the high-energy regime, where space charge effects can be neglected, the particles in the back of the bunch acquire a higher velocity than the ones in front, simply because they are accelerated over a longer distance. In the low-energy space charge 


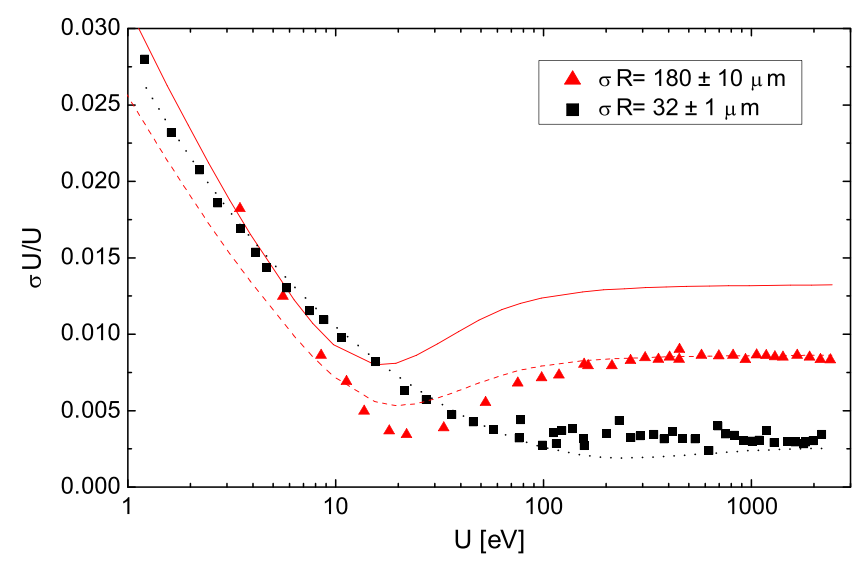

FIG. 4 (color online). Measurements (scatter plots) of $\sigma_{U} / U$ as function of $U$ for $\sigma_{R}=32$ and $180 \mu \mathrm{m}$, with, respectively, $Q=0.42 \mathrm{fC}$ and $Q \approx 0.5 \mathrm{fC}$. Also shown are simulation results for $\sigma_{R}=180 \mu \mathrm{m}$ (solid line), $\sigma_{R}=110 \mu \mathrm{m}$ (dashed line), $\sigma_{R}=32 \mu \mathrm{m}$ (dotted line).

dominated regime, on the other hand, the particles in the back acquire a lower velocity than the ones in front as they are decelerated by space charge forces. In the crossover regime these two effects cancel, leading to a further reduction of the relative energy spread. Related effects have been predicted in simulations of high-density electron bunch dynamics $[17,18]$. GPT calculations also show this behavior for large values of $\sigma_{R}$ (see Fig. 4). The agreement between simulation and experiments for $\sigma_{R}=180 \mu \mathrm{m}$ is not as good as for $\sigma_{R}=32 \mu \mathrm{m}$, which we attribute, at least partially, to distortion of the ionization laser beam profile. Further investigation is required to understand the detailed mechanism behind this subtle and possibly useful effect.

In summary, we have presented time-of-flight energy spread measurements on ion-bunches from an ultra cold ion source, which show that very low-energy-spread ion beams can indeed be produced. This result will certainly hold for continuous operation of the source, because space charge effects will be much less important at demonstrated currents of $1.4 \mathrm{pA}$ [9] and achievable currents of $100 \mathrm{pA}$ [4]. The lowest energy spread observed here, $\sigma_{U}=$ $0.02 \mathrm{eV}$, constitutes a 2 orders of magnitude improvement over the state-of-the-art liquid metal ion source. Welldefined, low-divergence, monochromatic beams with energies of only $1 \mathrm{eV}$ have been produced, creating entirely new possibilities for highly controlled ion-surface interaction, without sputtering damage. In addition, we have shown that the slowly moving ion bunches provide a model system for studying space charge effects in pulsed beams. The transition from space charge dominated dynamics to ballistic flight has been studied in great detail. Furthermore, a new effect has been observed; space charge induced energy spread reduction that could be used to lower the energy spread even further.
The authors would like to thank J. A.C. M. van de Ven, A. H. Kemper, L. H. A. M. van Moll, and H. A. van Doorn for their technical support during this project. This research is supported by the Dutch Technology Foundation STW, applied science division of the "Nederlandse Organisatie voor Wetenschappelijk Onderzoek (NWO)" and the Technology Program of the Ministry of Economic Affairs. This work is also part of the research program of the "Stichting voor Fundamenteel Onderzoek der Materie (FOM)," which is financially supported by NWO.

*O.J.Luiten@tue.nl

[1] J. Orloff, Rev. Sci. Instrum. 64, 1105 (1993).

[2] L. van Vaeck, A. Adriaens, and R. Gijbels, Mass Spectrom. Rev. 18, 1 (1999).

[3] J.S. Fletcher, A. Henderson, G.X. Biddulph, S. Vaidyanathan, N. P. Lockyer, and J. C. Vickerman, Appl. Surf. Sci. 255, 1264 (2008).

[4] S. B. van der Geer, M. P. Reijnders, M. J. de Loos, E. J. D. Vredenbregt, P. H. A. Mutsaers, and O. J. Luiten, J. Appl. Phys. 102, 094312 (2007).

[5] B. J. Claessens, S. B. van der Geer, G. Taban, E. J. D. Vredenbregt, and O. J. Luiten, Phys. Rev. Lett. 95, 164801 (2005).

[6] J. L. Hanssen, J. J. McClelland, E. A. Dakin, and M. Jacka, Phys. Rev. A 74, 063416 (2006).

[7] B. W. Ward, J. A. Notte, and N. P. Economou, J. Vac. Sci. Technol. B 24, 2871 (2006).

[8] B. J. Claessens, M. P. Reijnders, G. Taban, O. J. Luiten, and E. J.D. Vredenbregt, Phys. Plasmas 14, 093101 (2007).

[9] J.L. Hanssen, S. B. Hill, J. Orloff, and J. J. McClelland, Nano Lett. 8, 2844 (2008).

[10] P. G. OShea, M. Reiser, R. A. Kishek, S. Bernal, H. Li, M. Pruessner, V. Yun, Y. Cui, W. Zhang, Y. Zou, T. Godlove, D. Kehne, P. Haldemann, and I. Haber, Nucl. Instrum. Methods Phys. Res., Sect. A 464, 646 (2001).

[11] The Physics and Applications of High Brightness Electron Beams, edited by A. Zichichi (World Scientific, Singapore, 2005).

[12] H. J. Metcalf and P. van der Straten, Laser Cooling and Trapping (Springer, Berlin, 1999).

[13] G. Taban, M. P. Reijnders, S. C. Bell, S. B. van der Geer, O. J. Luiten, and E. J.D. Vredenbregt, Phys. Rev. ST Accel. Beams 11, 050102 (2008).

[14] http://www.pulsar.nl/gpt.

[15] J.H. Billen and L. M. Young, Los Alamos National Laboratory Report No. LA-UR-96-1834 (Poisson Superfish).

[16] J. Barnes and P. Hut, Nature (London) 324, 446 (1986).

[17] S. B. van der Geer, M. J. de Loos, T. van Oudheusden, W. P. E. M. op't Root, M. J. van der Wiel, and O. J. Luiten, Phys. Rev. ST Accel. Beams 9, 044203 (2006).

[18] D. F. Gordon, R. F. Hubbard, J. M. Cooley, B. Hafizi, A. Ting, and P. Sprangle, Phys. Rev. E 71, 026404 (2005). 